\title{
An Analytical Solution for Static Problems of Cantilever Curved Beams with Variable Cross Sections
}

\author{
I. ECSEDI ${ }^{1}$, Á. J. LENGYEL ${ }^{2}$, A. BAKSA ${ }^{3}$, D. GÖNCZI ${ }^{4}$ \\ 1,2,3,4 University of Miskolc, Institute of Applied Mechanics \\ ${ }^{1}$ mechecs@uni-miskolc.hu, ${ }^{2}$ mechlen@uni-miskolc.hu, ${ }^{3}$ mechab@uni-miskolc.hu, ${ }^{4}$ mechgoda@uni-miskolc.hu
}

Abstract. This paper gives an analytical method to obtain the deformation of a cantilever curved beam. The curved beam considered has circular centre line and the thickness of the cross section in radial direction depends on the circumferential coordinate. The kinematics of the Euler-Bernoulli beam model is used to formulate of governing equations. The curved homogeneous and isotropic elastic beam is fixed at the one of the end cross section and on the other end cross section is subjected to concentrated forces and a couple. A numerical example illustrates the applications of the derived formulae.

Keywords: Curved beam, cantilever, variable thickness, elastic, analytical

\section{Introduction}

The analysis of a curved beam is a standard topic in the most text-books of solid mechanics [1,2,3,4]. This theme is still concern at the present time because the curved elements are important components in many modern engineering structures. In this paper an analytical solution is presented for the cantilever curved beams whose thickness in radial direction is not constant. One of the end cross section of the curved beam is fixed and the other end cross section is subjected to radial and circumferential concentrated forces and a couple.

Elasticity solutions are presented in [5] for orthotropic functionally graded circular curved beam of uniform cross sections by means of Airy stress functions. The developed method is illustrated in curved cantilever beams with different types of loading conditions. Paper by Pydah and Sabale [6] presents an analytical model for the flexure of bidirectional functionally graded circular beam. The formulation of the considered problem is based on the Euler-Bernoulli's beam theory. The governing equations are solved for statically determinate circular cantilever beam under the action of tip loads. Paper [7] deals with the determination of stresses in circular curved beam of uniform cross section which has cross sectional inhomogeneity. In paper by Ecsedi and Lengyel [8] an analytical solution is given for the determination of deformations of curved composite beams with uniform cross sections. The developed analytical solution is based on fundamental solutions which are filling to the given loading and supporting conditions. Closed form formulae are derived for the displacements, cross-sectional rotation and the normal and shearing stresses. Several studies give finite element numerical solutions to the inplane deformation of static problems of circular curved beam with uniform cross sections such as $[9,10$, $11]$. 


\section{Governing equations}

In cylindrical coordinate system $\operatorname{Or} \varphi z$ the curved beam of variable cross section occupies the space domain $B$ (Fig. 1)

$$
B=\left\{(r, \varphi, z) \mid 0 \leq \varphi \leq \alpha, r_{1}(\varphi) \leq r \leq r_{2}(\varphi),-t / 2 \leq z \leq t / 2\right\},
$$

where $r_{i}(\varphi)(i=1,2)$ is the radius of the inner and of the outer cylindrical boundary surfaces of body $B$. $t$, which is constant, denotes the cross-sectional thickness along $z$ direction. The case, when the cross sectional thickness is not constant along the direction of axis $z$, is analysed in paper [13]. The radius $R$ of the circular centre line is also constant.
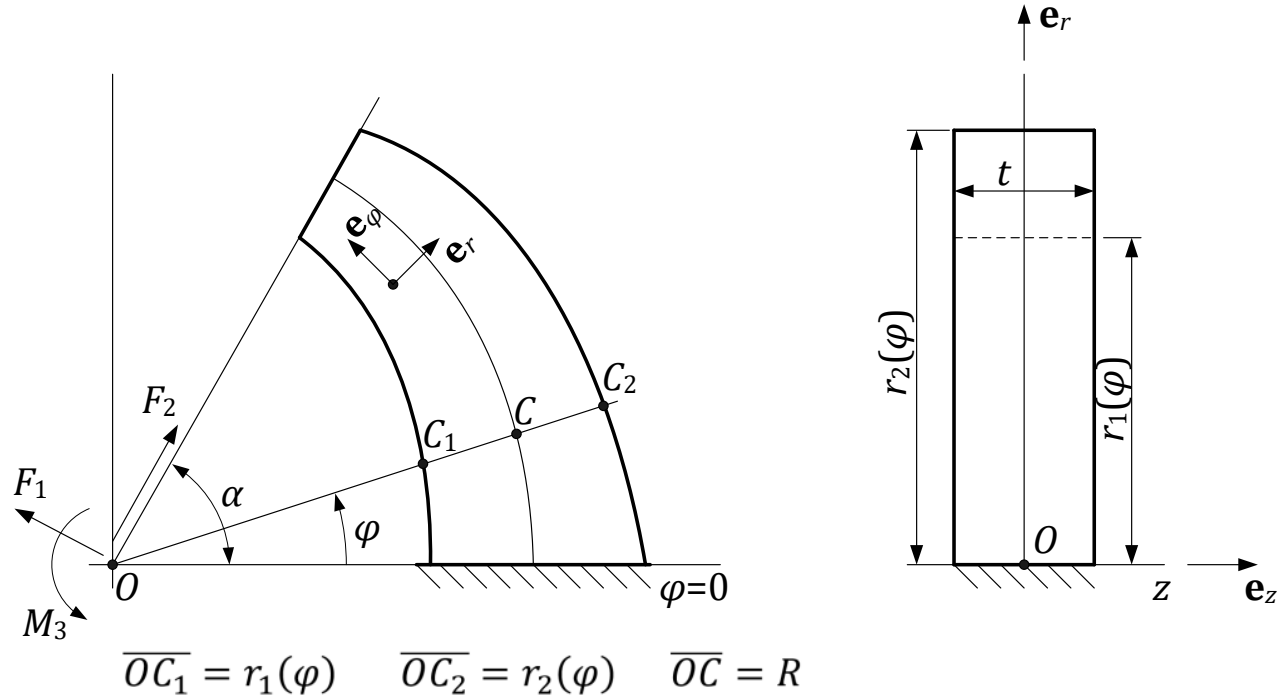

Figure 1. Cantilever curved beam with tip loads

The plane $z=0$ is the plane of symmetry of the curved beam and of the applied loads. Denote the unit vectors of cylindrical coordinate system $\operatorname{Or} \varphi z \mathbf{e}_{r}, \mathbf{e}_{\varphi}, \mathbf{e}_{z}$. The following displacement field is used to describe the in plane deformation of the cantilever beam [12]

$$
\mathbf{u}(r, \varphi)=U(\varphi) \mathbf{e}_{r}(\varphi)+(r \phi(\varphi)+V(\varphi)) \mathbf{e}_{\varphi}(\varphi), \quad V(\varphi)=\frac{\mathrm{d} U}{\mathrm{~d} \varphi} .
$$

Application of the strain-displacement relationships of the linearized theory of elasticity gives $[8,12]$

$$
\varepsilon_{r}=\varepsilon_{Z}=\gamma_{r z}=\gamma_{\varphi z}=\gamma_{r \varphi}=0, \quad \varepsilon_{\varphi}=\frac{W(\varphi)}{r}+\frac{\mathrm{d} \phi}{\mathrm{d} \varphi}, \quad W(\varphi)=\frac{\mathrm{d}^{2} U}{\mathrm{~d} \varphi^{2}}+U .
$$

In equation (3) $\varepsilon_{r}, \varepsilon_{\varphi}, \varepsilon_{z}$ are the longitudinal strains and $\gamma_{r \varphi}, \gamma_{r z}, \gamma_{z \varphi}$ are the shearing strains. The strains given by equation (3) satisfy the requirements of the Euler-Bernoulli's beam theory only the normal strain $\varepsilon_{\varphi}$ is different from zero and all the other strains vanish. According to papers $[8,12]$ we define the stress resultant forces $N=N(\varphi), S=S(\varphi)$ and stress couple resultant $M=M(\varphi)$ as

$$
N(\varphi)=\int_{A(\varphi)} \sigma_{\varphi}(r, \varphi) \mathrm{d} A, \quad S(\varphi)=\int_{A(\varphi)} \tau_{r \varphi}(r, \varphi) \mathrm{d} A, \quad M(\varphi)=\int_{A(\varphi)} r \sigma_{\varphi}(r, \varphi) \mathrm{d} A
$$

where $\tau_{r \varphi}=\tau_{r \varphi}(r, \varphi)$ denotes the shearing stress. Here, we note, the shear force $S=S(\varphi)$ will be computed by the use of a force equilibrium equation which is the usual way in the case of Euler-Bernoulli 
beam theory. Fig. 2 illustrates the stress resultant forces and the stress couple resultant in an arbitrary cross section of the curved beam.

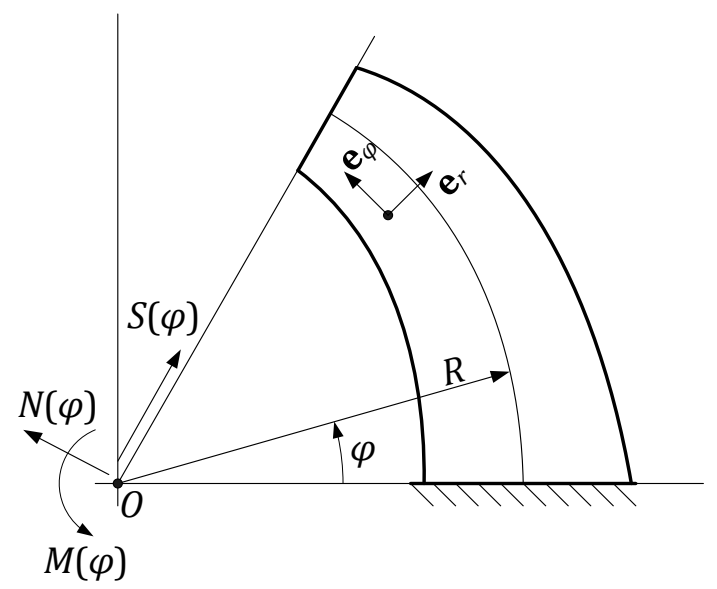

Figure 2. Illustration of $N(\varphi), S(\varphi)$ and $M(\varphi)$

Application of the Hooke's law gives

$$
\sigma_{\varphi}(r, \varphi)=E\left(\frac{W(\varphi)}{r}+\frac{\mathrm{d} \phi}{\mathrm{d} \varphi}\right)
$$

where $E$ is the modulus of elasticity. From equation (4) it follows that

$$
N(\varphi)=E t\left(a(\varphi) W(\varphi)+b(\varphi) \frac{\mathrm{d} \phi}{\mathrm{d} \varphi}\right), \quad M(\varphi)=E t b(\varphi)\left(W(\varphi)+R \frac{\mathrm{d} \phi}{\mathrm{d} \varphi}\right)
$$

Here,

$$
a(\varphi)=\ln \left(\frac{r_{2}(\varphi)}{r_{1}(\varphi)}\right), \quad b(\varphi)=r_{2}(\varphi)-r_{1}(\varphi)
$$

The equilibrium equations in terms of $N, S$ and $M$ for circular curved cantilever beam which is loaded only its end cross sections are $[8,12]$

$$
\frac{\mathrm{d} N}{\mathrm{~d} \varphi}+S=0, \quad \frac{\mathrm{d} S}{\mathrm{~d} \varphi}-N=0, \quad \frac{\mathrm{d} M}{\mathrm{~d} \varphi}=0 .
$$

We remark that the considered cantilever curved beams satisfy

$$
U(0)=0, \quad V(0)=0, \quad \phi(0)=0 .
$$

Fig. 1 shows the curved cantilever beam with the applied load system. The solution of equilibrium equations (8) can be represented as

$N(\varphi)=-F_{1} \sin (\varphi-\alpha)+F_{2} \cos (\varphi-\alpha), \quad S(\varphi)=F_{1} \cos (\varphi-\alpha)+F_{2} \sin (\varphi-\alpha), \quad M(\varphi)=M_{3}$.

\section{Formulation of the analytical solution}

From equation (6) we can derive a system of linear equations for $W(\varphi)$ and $\phi_{1}(\varphi)=\frac{\mathrm{d} \phi}{\mathrm{d} \varphi}$

$$
\begin{gathered}
a(\varphi) W(\varphi)+b(\varphi) \phi_{1}(\varphi)=\frac{1}{E t}\left(-F_{1} \sin (\varphi-\alpha)+F_{2} \cos (\varphi-\alpha)\right), \\
W(\varphi)+R \phi_{1}(\varphi)=\frac{M_{3}}{E t b(\varphi)} .
\end{gathered}
$$


The solution of the system of linear equations is as follows

$$
\begin{gathered}
W(\varphi)=\frac{R}{E t} \frac{N(\varphi)}{R a(\varphi)-b(\varphi)}-\frac{1}{E t} \frac{M_{3}}{R a(\varphi)-b(\varphi)}, \\
\phi_{1}(\varphi)=-\frac{1}{E t} \frac{N(\varphi)}{R a(\varphi)-b(\varphi)}+\frac{1}{E t} \frac{a(\varphi)}{b(\varphi)} \frac{M_{3}}{R a(\varphi)-b(\varphi)} .
\end{gathered}
$$

Here, the undermentioned result of the theory of ordinary differential equations will be used [14, 15].

Theorem. Let $h=h(x)$ be a continuous bounded function defined for $x \in[0, a>0]$. In this case the solution of the initial-value problem

$$
\frac{\mathrm{d}^{2} y}{\mathrm{~d} y^{2}}+y=h(x), \quad 0 \leq x \leq a, \quad y(0)=0, \quad\left(\frac{\mathrm{d} y}{\mathrm{~d} x}\right)_{x=0}=0
$$

can be represented as

$$
y(x)=\int_{0}^{x} h(\lambda) \sin (x-\lambda) \mathrm{d} \lambda
$$

and we have

$$
\frac{\mathrm{d} y}{\mathrm{~d} x}=\int_{0}^{x} h(\lambda) \cos (x-\lambda) \mathrm{d} \lambda .
$$

The application of cited Theorem gives the formulae of the radial displacement $U(\varphi)=U_{1}(\varphi)+U_{2}(\varphi)$ and circumferential displacement $V(\varphi)=V_{1}(\varphi)+V_{2}(\varphi)$

$$
\begin{gathered}
U_{1}(\varphi)=\frac{R}{E t} \int_{0}^{\varphi} \frac{F_{2} \cos (\vartheta-\alpha)-F_{1} \sin (\vartheta-\alpha)}{R a(\vartheta)-b(\vartheta)} \sin (\varphi-\vartheta) \mathrm{d} \vartheta, \\
U_{2}(\varphi)=-\frac{M_{3}}{E t} \int_{0}^{\varphi} \frac{\sin (\varphi-\vartheta)}{R a(\vartheta)-b(\vartheta)} \mathrm{d} \vartheta, \\
V_{1}(\varphi)=\frac{R}{E t} \int_{0}^{\varphi} \frac{F_{2} \cos (\vartheta-\alpha)-F_{1} \sin (\vartheta-\alpha)}{R a(\vartheta)-b(\vartheta)} \cos (\varphi-\vartheta) \mathrm{d} \vartheta, \\
V_{2}(\varphi)=-\frac{M_{3}}{E t} \int_{0}^{\varphi} \frac{\cos (\varphi-\vartheta)}{R a(\vartheta)-b(\vartheta)} \mathrm{d} \vartheta .
\end{gathered}
$$

The expression of the cross-sectional rotation $\phi=\phi(\varphi)$ can be obtained by integration of equation (14)

$$
\phi(\varphi)=\frac{1}{E t}\left\{\int_{0}^{\varphi}\left[\frac{F_{1} \sin (\vartheta-\alpha)-F_{2} \cos (\vartheta-\alpha)}{R a(\vartheta)-b(\vartheta)}+\frac{M_{3} a(\vartheta)}{b(\vartheta)(R a(\vartheta)-b(\vartheta))}\right] \mathrm{d} \vartheta\right\},
$$

The determination of the expression of the circumferential normal stress $\sigma_{\varphi}(r, \varphi)$ is based on equation (5) by a brief computation we get that

$$
\sigma_{\varphi}(r, \varphi)=\frac{1}{t(R a(\varphi)-b(\varphi))}\left\{F_{1}\left(1-\frac{R}{r}\right) \sin (\varphi-\alpha)-F_{2}\left(1-\frac{R}{r}\right) \cos (\varphi-\alpha)+M_{3}\left(\frac{a(\varphi)}{b(\varphi)}-\frac{1}{r}\right)\right\} .
$$

From the formula (23) it follows that if $M_{3}=0$ then we have $\sigma_{\varphi}(R, \varphi)=0$ for $0 \leq \varphi \leq \alpha$.

\section{Numerical example}

The following data are used in the numerical example: $R=60 \mathrm{~mm}, t=3 \mathrm{~mm}, \alpha=\pi / 2$, $E=2 \times 10^{5} \frac{\mathrm{N}}{\mathrm{m}^{2}}, \beta=0.25$, 


$$
r_{1}(\varphi)=R\left[1-\frac{0.25 \beta}{\varphi+\frac{\pi}{20}}\right], \quad r_{2}(\varphi)=R\left[1+\frac{0.25 \beta}{\varphi+\frac{\pi}{20}}\right]
$$

The graph of the radial displacement $U=U(\varphi)$ and the graph of the circumferential displacement $V=V(\varphi)$ are shown in Fig.3 and Fig.4, respectively. In Fig. 5, the plot of the cross-sectional rotation $\phi=\phi(\varphi)$ is presented as a function of $\varphi$.

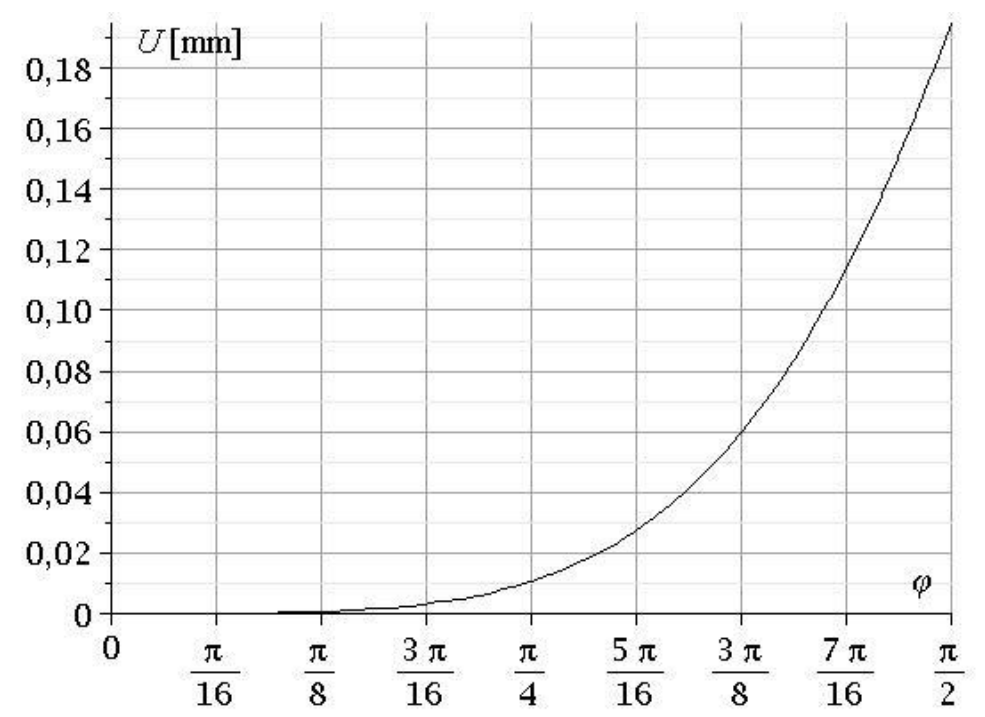

Figure 3. Plot of $U=U(\varphi)$

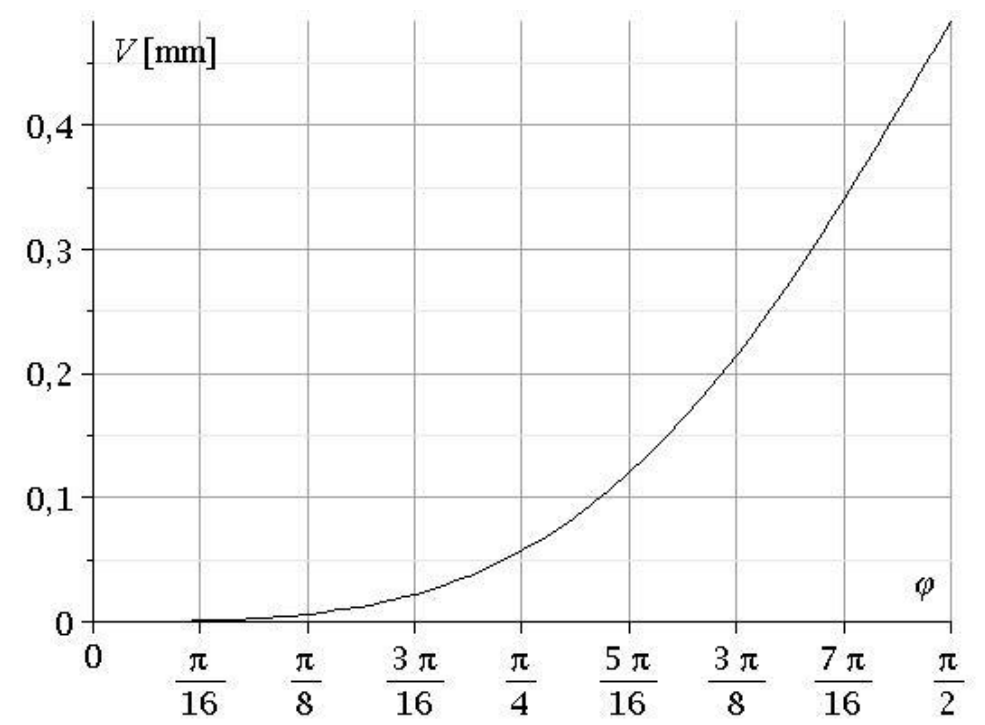

Figure 4. Plot of $V=V(\varphi)$ 


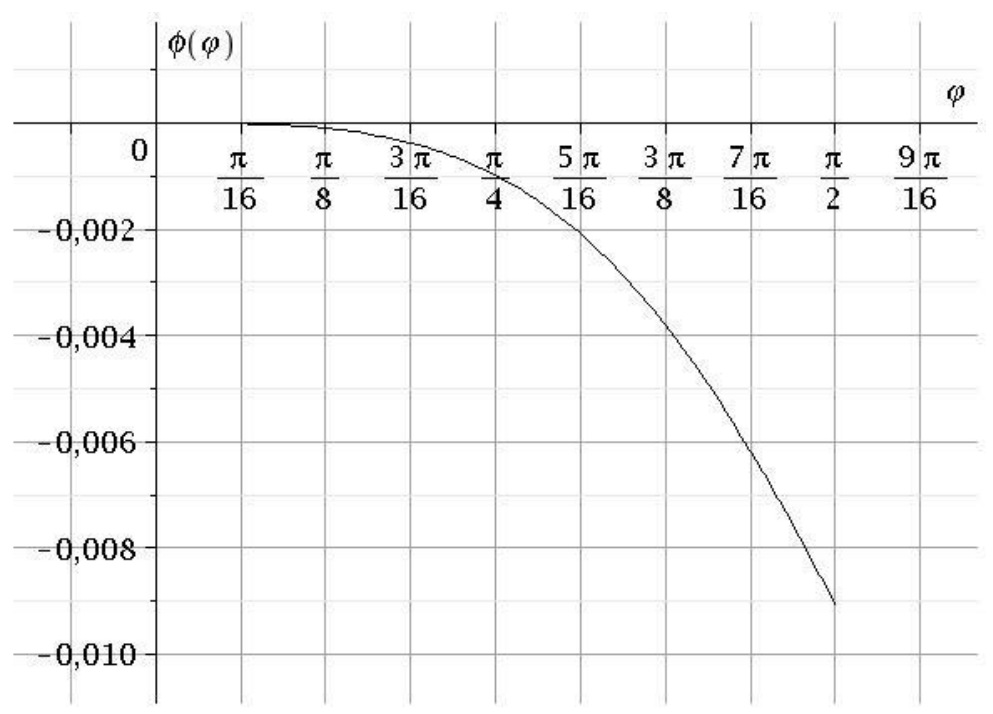

Figure 5. Plot of $\phi=\phi(\varphi)$

The plots of $\sigma_{\varphi}(r, \varphi)$ for three different values of polar angle $\varphi\left(\varphi=0, \varphi=\frac{\pi}{6}, \varphi=\alpha=\frac{\pi}{2}\right)$ are shown in Fig. 6. Fig. 7 gives the distribution of the normal stress in the fixed end cross section, $\varphi=0$.

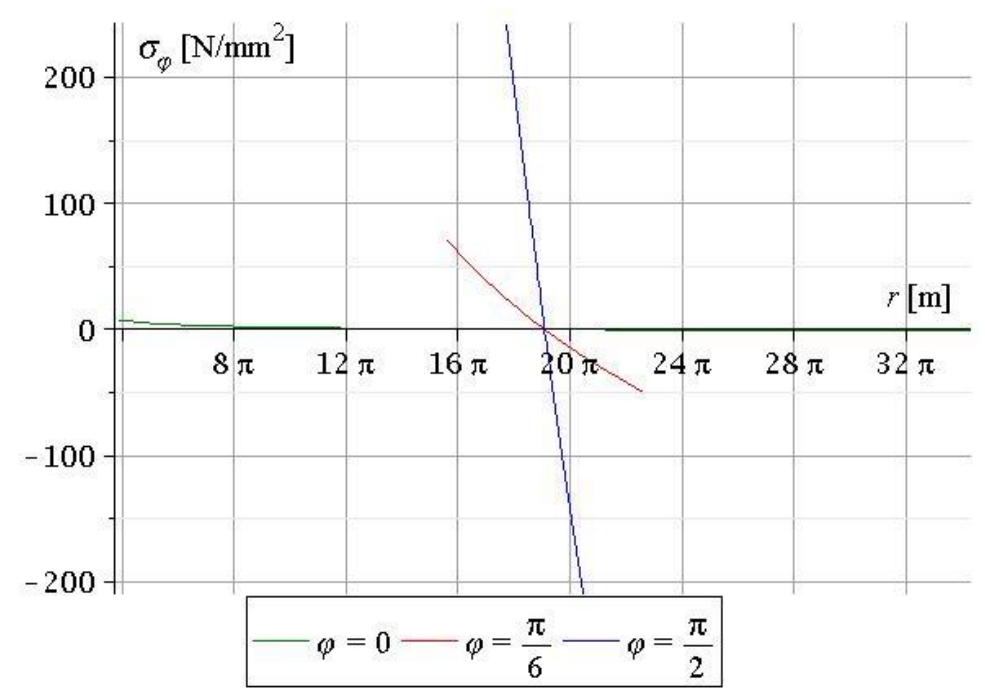

Figure 6. The plots of $\sigma_{\varphi}(r, \varphi)$ for $\varphi=0, \varphi=\frac{\pi}{6}, \varphi=\alpha=\frac{\pi}{2}$ 


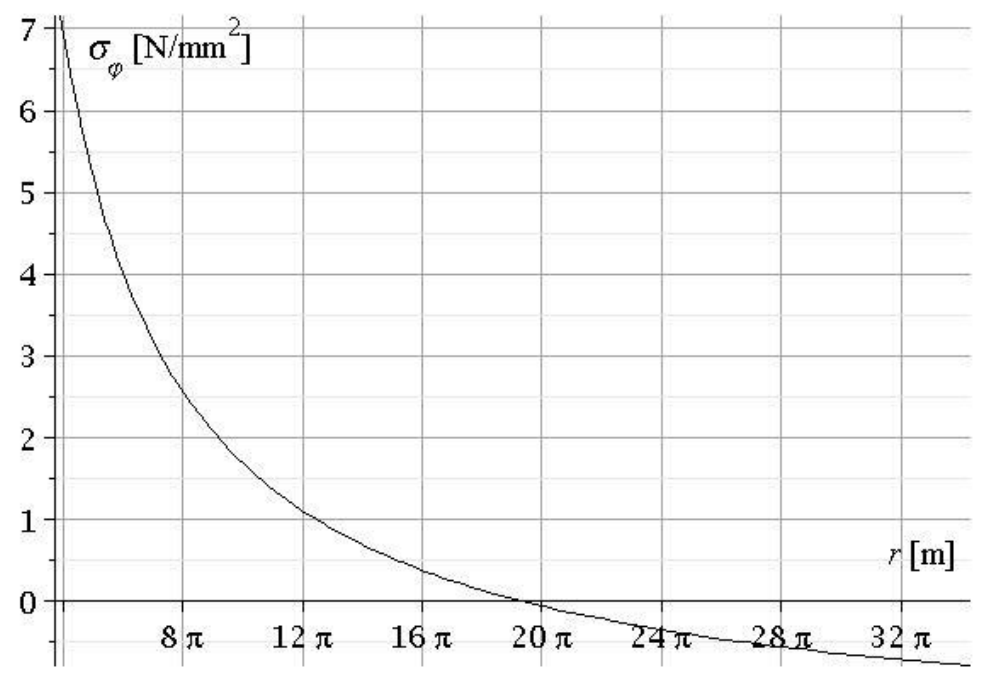

Figure 7. The graph of $\sigma_{\varphi}(r, 0)$

\section{Conclusions}

In this paper elastic cantilever curved beam of variable cross section is studied. The thickness of the curved circular beam in radial direction is not constant it is a smooth function of the polar angle. The considered curved beam at one of the end cross section is fixed and at the other end cross section is loaded by two concentrated forces and a couple. Paper presents an analytical solution of the above mentioned statics problem. The formulation is based on the Euler-Bernoulli curved beam theory. The results of the presented numerical example can be used as a benchmark solution to verify the accuracy of the results obtained by other methods.

\section{References}

[1] Boresi, A. P., Schmidt, R. J., Sidebottom, O. M. (2003) 'Advanced Mechanics of Materials', New York: Wiley and Sons Inc., doi: 10.1111/j.1475-1305.1993.tb00852.x

[2] Solecki, R., Conant, R. J. (2003) 'Advanced Mechanics of Materials'. Oxford: Oxford University Press. ISBN 978-0195143720

[3] Cook, R. D., Young, W. C. (1985) 'Advanced Mechanics of Materials'. New York: McMillan. ISBN 9780023246203

[4] Saada, A. S. (1974) 'Elasticity. Theory and its Applications' London: Pergamon Press Inc., doi: 10.1016/C2013-0-02524-8

[5] Wang, M., Liu, Y. (2013) 'Elasticity solutions for orthotropic functionally graded curved beams', European Journal of Mechanics - A/Solids, 37, pp. 8-16.

doi: 10.1016/j.euromechsol.2012.04.005

[6] Pydah, A., Sabale, A. (2017) 'Static analysis of bi-directional functionally graded curved beams', Composite Structures, 160, pp. 867-876. doi: 10.1016/j.compstruct.2016.10.120 
[7] Kiss, L., Szeidl, Gy. (2015) 'Stresses in curved beams made of heterogeneous materials', International Journal of Mechanical Systems Engineering, 1(2), p. 8. article id 1:IJMSE-107. doi: 10.15344/2455-7412/2015/107

[8] Ecsedi, I., Lengyel, Á. J. (2019) 'An analytical solution for static problems of curved composite beams', Curved and Layered Structures, 6(1), pp. 105-116. doi: 10.1515/cls-2019-0009

[9] Ascione, L., Fraternali, F. (1992) 'A penalty model for the analysis of curved composite beams', Computers and Structures, 45(5-6), pp. 985-999. doi: 10.1016/0045-7949(92)90057-7

[10] Ibrahimbegović, A., Frey, F. (1993) "Finite element analysis of linear and non-linear planar deformation of elastic initially curved beams', International Journal for Numerical Methods in Engineering, 36(19), pp. 3239-3258. doi: 10.1002/nme.1620361903

[11] Kim, J. G. (2005) 'An effective composite laminated curved beam element', Communications in Numerical Methods in Engineering, 22(5), pp. 453-466. doi: 10.1002/cnm.829

[12] Ecsedi, I., Dluhi, K. (2005) 'A linear model for the static and dynamic analysis of nonhomogeneous curved beams.' Applied Mathematical Modelling, 29(12), pp. 1211-1231. doi: 10.1016/j.apm.2005.03.006

[13] Ecsedi, I., Baksa, A. (2021) 'Deformation of cantilever curved beam with variable cross section', Journal of Computational and Applied Mechanics, 16(1), pp. 23-36, doi: $10.32973 /$ jcam.2021.002

[14] Kamke, E. (1951) 'Differentialgleichungen. Lösungsmethoden und Lösungen I-II.' Leipzig: Akademische Verlagsgesellschaft Gest and Portig K.-G., ISBN: 978-3663120582

[15] Forsyth, A. R. (1929) 'A Treatise on Differential Equations.' London: 6th Ed., MacMillan. 